\title{
A Case of 25 Year Old Dwarf with Classic Cockayne Syndrome
}

\author{
MOHAMMED FR ${ }^{1}$, CHOWDHURY FR ${ }^{1}$, NUR $Z^{1}$, SHAMS MZ², ALAM MB ${ }^{3}$, AHASAN HAMN ${ }^{4}$
}

\begin{abstract}
A 25 year old, short stature man of non-consanguineous parents, attended with acute respiratory tract infection along with progressive difficulties in walking, hearing and vision. He had the complaints of growth retardation, poor feeding, listless attitude and delayed milestones of development since one year of age. At presentation, he was non cooperative, IQ below 50; height and weight were below $5^{\text {th }}$ percentile. He had progeria with enophthalmos, cataract, corneal opacity, miotic pupils, tremor, ataxia, in-coordination of movement, diminished tendon reflexes, unsteady gait, bilateral sensory neural deafness and hepatomegaly. Lateral skull X-ray showed cortical calcification. MRI of brain revealed bilateral dentate nucleus and basal ganglia calcification, generalized cerebral and cerebellar atrophy and ventricular dilatation. Typical clinical and imaging findings clinched the diagnosis of classic Cockayne Syndrome; which is a rare, autosomal recessive, DNA repair deficient, multisystem disorder. It has no cure and the prognosis is poor.
\end{abstract}

Keyword: Classic Cockayne syndrome, Dwarfism, Basal ganglia calcification, Cerebral atrophy

\section{Introduction}

Cockayne Syndrome (CS), also known as Neill-Dingwall Syndrome, Progeroid Nanism, Deafness-Dwarfism-Retinal Atrophy, is a rare form of dwarfism with an autosomal recessive inheritance. ${ }^{1}$ It is a DNA repair deficient multisystem disorder characterized principally by growth retardation, photosensitivity and premature aging (progeria). ${ }^{1,2}$ Cockayne syndrome comprises a spectrum of disorders. For practical purpose four classes are proposed. These are the "classic" form (CS type I); a more severe "Congenital” form (CS type II), a "Late Onset” form (CS type III) and xeroderma pigmentosum-Cockayne syndrome (XPCS). ${ }^{3}$ Fetal growth is normal in classic CS and signs, symptoms appear around the first two years of life. Death usually occurs in the first or second decade of life due to progressive and unremitting degeneration of the central and peripheral nervous system. CS type II or "connatal" CS is also known as cerebro-oculo-facial syndrome (COFS) or PenaShokeir syndrome type II. As the name implies; it is characterized by intrauterine growth retardation, little or no postnatal neurologic development, postnatal contractures of the spine and death occurs earlier by the age of seven years. Characteristic facial phenotype of CS, CNS degeneration and skeletal abnormalities are absent in case of XP-CS. ${ }^{3}$ Fortunately, skin cancer is not reported in Cockayne syndrome, despite photosensitivity and DNA repair deficiency. ${ }^{2}$ Hitherto, we depict a case of a 25 year old dwarf who first attended the adult ward and was found to satisfy the criteria of classic CS.

\section{Case report}

A 25 year old short stature male attended to us with acute respiratory tract infection along with progressive difficulties in walking, hearing and vision. Further query revealed that he had an eventful history since his infancy. He is the eldest son of nonconsanguineous parents. His mother was 20 years at the time of his birth and he was born at term after an uneventful pregnancy. His birth weight was $2.6 \mathrm{~kg}$ and he had been thriving well up to one year of age. Thereafter his growth started to cease day by day with delayed milestones of development. He learned to walk at one year. But he had to seek help for another year to walk alone and his gait is unsteady still now. He was reluctant to take food. He never achieved weight and height above $3^{\text {rd }}$ percentile according to age. He had slurring of speech and gradually became apathic. He could not communicate with others reasonably according to his age since his childhood. He failed to cope with customary classroom setting and therefore remained illiterate. By the age of 15 he noticed visual difficulty, photophobia and impaired hearing. These symptoms were progressive and at present he can hear very little and is blind. He attained puberty lately at 20 years. He has no family history of such illness and all his siblings are in good health.

1. Post Graduate Trainee, Department of Medicine, Dhaka Medical College Hospital, Dhaka.

2. Assistant Registrar, Department of Medicine, Dhaka Medical College Hospital, Dhaka.

3. Associate Professor, Department of Medicine, Dhaka Medical College, Dhaka.

4. Professor, Department of Medicine, Dhaka Medical College, Dhaka.

Correspondence: Dr. Fazle Rabbi Mohammed, Flat-C1, House no-29, Road no-5, Dhanmondi, Dhaka-1205, Bangladesh. E-mail: rabbimd@gmail.com 
On examination we found him non cooperative, IQ below 50, length of upper limbs $52 \mathrm{~cm}$ and lower limbs $56 \mathrm{~cm}$. His head circumference was $46 \mathrm{~cm}$, height was 1.12 meter and weight was $17 \mathrm{~kg}$. All these parameters are much below $5^{\text {th }}$ percentile according to his age. He had a senile appearance with sunken eyes and dry skin and hair (figure 1). His blood pressure was 110/70 mmHg and other vital signs were normal. Cataract, corneal opacity and poor papillary dilation were identified in both eyes but especially in the right one. His higher psychic

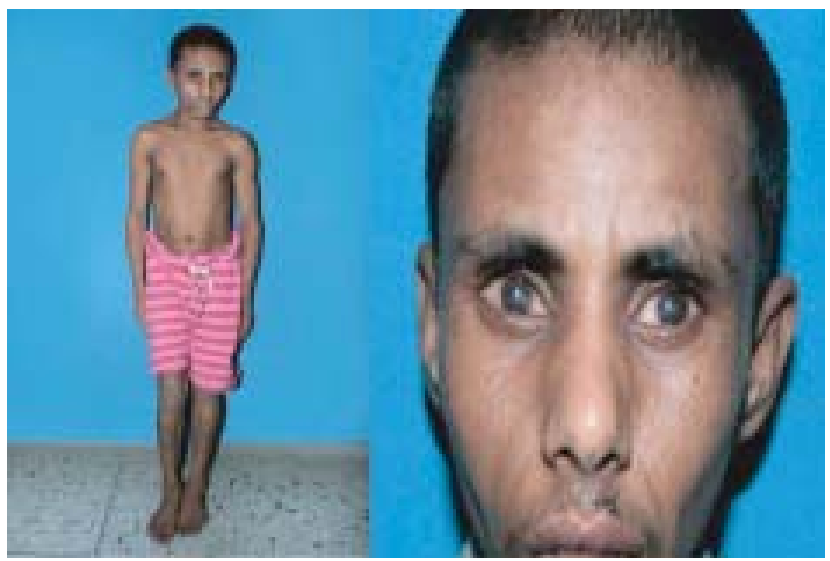

(a)

(b)

Fig.-1: A characteristic physical appearance of "cachectic dwarfism" with a stooped standing posture (a). The characteristic facies with lack of subcutaneous facial fat, marked enophthalmos and a beaked nose (b).

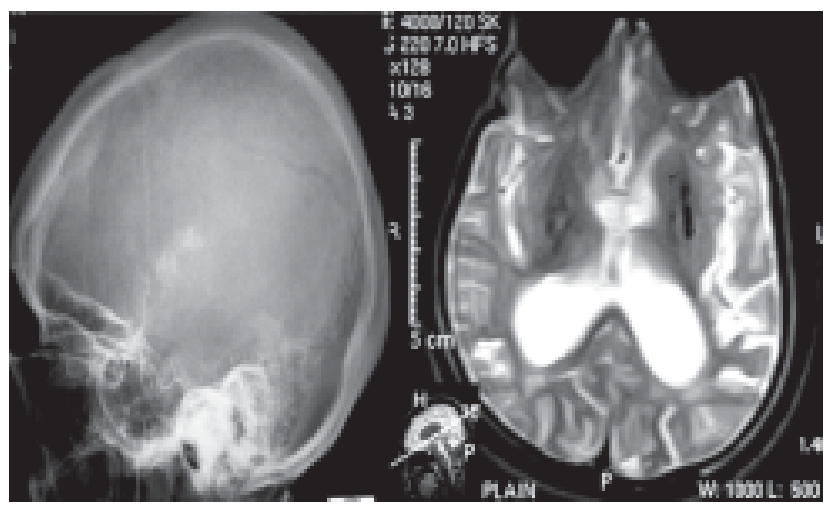

(a) function was impaired with a mini mental test score of 12 out of 30. Bulk of muscles was average according to height with normal muscle tone and power. All deep tendon reflexes were markedly diminished and planter reflexes were bilaterally flexor without any sensory impairment. He had action tremor, ataxia, in-coordination of movement, unsteady limping gait and bilateral sensory neural hearing loss. Mild hepatomegaly was present. Primary sexual organs were well developed according to his height and age with normal male pattern pubic hair and moustaches.

On investigation, complete blood count, serum creatinine, serum T3, T4, TSH and Prolactin level were found within normal limit. Serum Calcium was $2.5 \mathrm{mmol} / \mathrm{L}$, Parathyroid hormone level was 9.11pgm/ml and Alanine Aminotransferase (ALT) level was 120 IU/L. Ultra sonogram of whole abdomen revealed mild hepatomegaly. Pure tone audiogram showed bilateral sensory neural deafness. $\mathrm{X}$ ray of both hands revealed that all bones were proportionate but shorter according to age and all the growth ends were fused. X ray skull lateral view showed an area of calcification in mid cortex. MRI of brain revealed bilateral calcification of basal ganglia and dentate nucleus. Generalized cerebral and cerebellar atrophy and ventricular dilatation were also observed (Figure 2). Genetic analysis for mutation is arranged on a research basis only and expertise in this field is limited. As the boy nicely fit the frame of classic Cockayne syndrome, chromosome analysis was not done for diagnostic purpose.

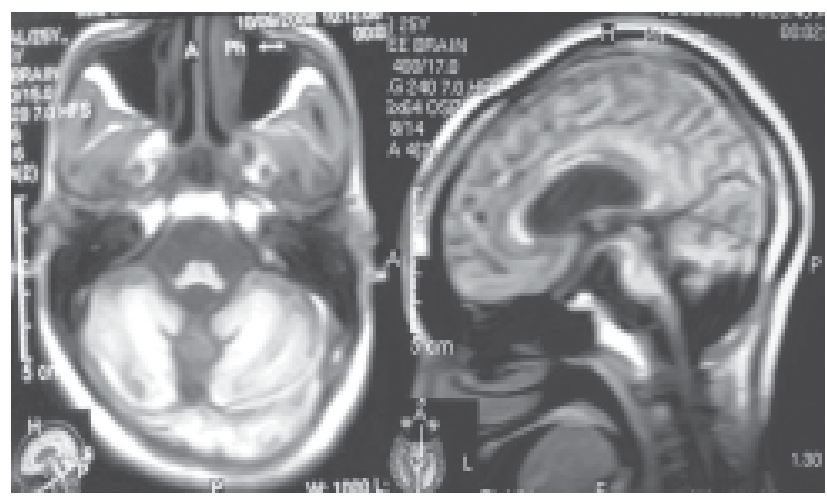

(c) (d)

Fig.-2: Radiological imaging of the patient. Lateral skull $x$-ray shows radio-opaque area indicating calcium deposition at the level of basal ganglia and calvarial thickening (a). An axial $T_{2}$-weighted image demonstrates areas of low intensity in the basal ganglia reflecting calcification. Generalized brain atrophy as well as atrophy of internal capsule is also seen (b). An axial FLAIR image showing calcification of cerebellum including dentate nucleus (c). A sagittal T1-weighted image demonstrates generalized atrophy of cerebrum, cerebellum and corpus callosum. Hypointensity at the cerebellum is also observed (d). 


\section{Discussion}

Edward Alfred Cockayne (1880-1956), a renowned London based physician, first recognized this syndrome (which was later uttered by his name). ${ }^{5}$ It was first reported in 1936 and the prevalence is unknown. ${ }^{3,4}$ Because, reports are available only as single case or family profiles. It probably occurs in less than 1 in 100,000 individuals. ${ }^{3}$ No sexual or racial predilection is reported yet. Mutations in two genes are found to be responsible for the disorder, ERCC6(CKN1) (75\% of individuals) and ERCC8 (25\% of individuals), located on chromosomes 5 and 10 respectively. ${ }^{2,6}$ Either gene failed to show any obvious genotype-phenotype correlation. Therefore, gene mutation alone cannot explain the clinical variability within the spectrum of CS. ${ }^{7,8}$ Diagnosis of classic Cockayne syndrome is clean cut when both the major criteria and three minor criteria are evident in an older child. ${ }^{3}$ Major criteria include postnatal growth failure (height and weight $<5$ th percentile by age 2 years) and progressive neurologic dysfunction. In most of the cases neurologic degeneration is characterized by early developmental delay. Leukodystrophy and intracranial calcifications in brain MRI and progressive behavioral and intellectual abnormalities are always observed. Seven minor criteria include a characteristic physical appearance of "cachectic dwarfism"; cutaneous photosensitivity with or without thin or dry skin or hair; demyelinating peripheral neuropathy; sensorineural hearing loss; pigmentary retinopathy and/or cataracts; characteristic radiographic findings of thickening of the calvarium, sclerotic epiphyses, vertebral and pelvic abnormalities; dental caries. ${ }^{3}$ Other reported findings are microcephaly, hyporeflexia, abnormal gait or inability to walk, ataxia, tremor, nystagmus, abnormal speech, poor feeding, delayed or absent sexual maturation, elevated liver enzymes, hepatomegaly, splenomegaly etc. ${ }^{3,4}$ Molecular genetic testing might be considered for atypical cases. ${ }^{3}$ Prenatal diagnosis is possible by amniocentesis. ${ }^{4}$ The clinical findings in our patient satisfy both the major criteria and most of the minor criteria and permit us to establish a diagnosis of classic Cockayne syndrome. Moreover, the typical imaging findings aid to wrap up the diagnosis.

Cockayne syndrome is not yet curable with a poor prognosis. Supportive measures to relieve symptoms and management of complications are mainstay of treatment. Multidisciplinary approach is essential. Occupational and physiotherapy are required for rehabilitation. Sunlight should be avoided and proper clothing and sunscreen should be used to protect cell damage and cataract formation. Insertion of feeding gastrostomy tube may prevent growth failure and malnutrition. Drugs can be used to ease spasticity. ${ }^{3}$ Cochlear implantation improves deafness. ${ }^{9}$ We provided him physiotherapy, symptomatic treatment along with necessary advices. He is on regular monthly follow up. Although the disorder seldom attacks, the affected persons and their families go through endless agony. Thus, genetic counseling is crucial to avoid this type of catastrophe in future.

\section{Conflict of interest: None}

\section{References}

1. National Organization for Rare Disorders. Cockayne Syndrome, 2008. Available at: http://www.rarediseases.org/ search/rdbdetail_abstract.html?disname=Cockayne $\% 20$ Syndrome. Accessed March 23, 2009.

2. Neilan EG. Cockayne Syndrome. In: Gene Reviews [online]. Available at: http://www.ncbi.nlm.nih.gov/bookshelf/ br.fcgi?book=gene\&part=cockayne. Accessed March 23, 2009.

3. Kraemer KH, Patronas NJ, Schiffmann R, et al. Xeroderma pigmentosum, trichothiodystrophy and Cockayne syndrome: a complex genotype-phenotype relationship. Neuroscience 2007; 145(4):1388-96.

4. Share \& Care Cockayne Syndrome Network. About CS, 2009. Available at: http://www.cockaynesyndrome.net/main/ AboutCS/tabid/54/Default.aspx. Accessed March 23, 2009.

5. Stefanini M, Fawcett H, Botta E, Nardo T, Lehmann AR. Genetic analysis of twenty-two patients with Cockayne syndrome. Hum Genet 1996; 97: 418-23.

6. Rapin I, Lindenbaum Y, Dickson DW, Kraemer KH, Robbins JH. Cockayne syndrome and xeroderma pigmentosum. Neurology 2000; 55: 1442-9.

7. Horibata K, Iwamoto Y, Kuraoka I, et al. Complete absence of Cockayne syndrome group $\mathrm{B}$ gene product gives rise to UV-sensitive syndrome but not Cockayne syndrome. Proc Natl Acad Sci USA 2004; 101: 15410-5.

8. Imaeda S. Cockayne Syndrome. In: eMedicine Specialties > Dermatology > Pediatric Diseases, 2007. Available at: http:/ /emedicine.medscape.com/article/1115866-overview. Accessed March 23, 2009.

9. Morris DP, Alian W, Maessen H, et al. Cochlear implantation in Cockayne syndrome: our experience of two cases with different outcomes. Laryngoscope 2007; 117(5):939-43. 